\title{
CONSTRUYENDO AL FUTURO SER SOCIAL: INTERVENCIONES MÉDICAS Y PEDAGÓGICAS EN LA INFANCIA ANORMAL. SANTIAGO DE CHILE, 1920-1943*
}

\author{
Marco Antonio León León \\ Universidad del Bío Bío / Universidad de Concepción \\ marcoaleon@hotmail.com \\ Mauricio Rojas Gómez \\ Universidad del Bío Bío / Universidad de Concepción \\ mrojas@ubiobio.cl
}

Recibido: 18 noviembre 2014; Aceptado: 6 febrero 2015.

Cómo citar este artículo/Citation: León León, Marco Antonio y Rojas Gómez, Mauricio (2015), “Construyendo al futuro ser social: Intervenciones médicas y pedagógicas en la infancia anormal. Santiago de Chile, 1920-1943", Asclepio, 67 (2): p114. doi: http:// dx.doi.org/10.3989/asclepio.2015.32

RESUMEN: El presente trabajo busca estudiar la relación entre el Estado y la anormalidad en Santiago de Chile a través de una categoría específica como fue la infancia anormal, que tanto en el plano discursivo como institucional, tomó mayor definición y organización desde inicios de la década de 1920 hasta mediados de los años 40. Más allá de los cambios en los criterios estatales que tomaron lugar, se puede apreciar la pervivencia de lógicas preventivas y de control junto a otras protectoras y asistenciales visibles en la legislación y las instituciones creadas. Se destaca el papel de la medicina y la pedagogía en este ámbito, y en particular el papel de la última en la generación de una ciudadanía asimilable a los proyectos industriales y de nación de la época.

PALABRAS CLAVE: Estado; Anormalidad; Medicina; Pedagogía; Inseguridad.

\section{BUILDING THE FUTURE "SOCIAL BEING": MEDICAL AND EDUCATIONAL INTERVENTIONS IN ABNOR-} MAL CHILDREN. SANTIAGO DE CHILE, 1920-1943

ABSTRACT: This paper seeks to explore the relationship between the State and abnormality in Santiago of Chile through a specific category as it was the abnormal childhood, both in the discursive and institutional levels, took greater definition and organization since the beginning of the decade of 1920 until the mid-1940s. Apart from changes in the State criteria that took place, you can see the survival of preventive logic and control along with other protective and assistance on legislation and the institutions created. Highlights the role of medicine and pedagogy in this area, and in particular the role of this last in the generation of an assimilable citizenship to the industrial and nation projects.

KEY WORDS: State, Abnormality; Medicine; Education; Insecurity. 


\section{INTRODUCCION: ESTADO Y ANORMALIDAD}

La primera mitad del siglo XX chileno se encuentra atravesada por múltiples problemáticas que superan los aspectos políticos y sociales generalmente estudiados (Correa et. al., 2001. Illanes, 2007. Salazar y Pinto, 1999. Yáñez, 2008). No se trata sólo de especificar una modificación en el régimen de gobierno (de Parlamentario a Presidencial) o la aparición de una nueva Constitución en 1925, sino también de establecer la existencia de dos modelos de Estado entrelazados que poco a poco irán presentando mutaciones y redefiniciones. Uno de ellos, basado en la prevención, la vigilancia y el control (Defensa Social) y el otro, en la protección y la intervención social (Asistencialista), criterios que no serán contradictorios, sino más bien complementarios, pues en particular, desde la década de 1930 en adelante, los conceptos aquí anotados se vincularán en más de una ocasión para definir políticas públicas y tomar medidas legislativas e institucionales destinadas a concretar el bienestar futuro de la población. Tal proceso, no significó un cambio automático ni lineal, por lo cual abordarlo debidamente no sólo demuestra su complejidad, sino asimismo, la necesidad de entender que una época histórica debe ser estudiada a través de diversas variables y no tan sólo desde una perspectiva.

Una categoría que muestra tanto la complejidad y el entrelazamiento de los conceptos aludidos es la anormalidad, la cual no era nueva dentro del período en estudio, pues encontraba sus orígenes en teorías deterministas y degenerativas vigentes desde el siglo XIX (Álvarez Uría, 1991 y 1996. Huertas, 1998 y 2005), pero que volvían a tomar fuerza en un contexto que propiciaba un nuevo papel del Estado como garante de la educación, la seguridad y la inclusión social (León, 2003-2004). En tal perspectiva, lo normal era vinculado a la ciencia, la racionalidad y el orden, que aparecían como vías legitimadoras de determinadas formas de poder; mientras que su contracara, lo anormal, era expuesta como una muestra de barbarie, irracionalidad y monstruosidad; pues connotaba carencias (deficiencias) físicas, mentales y morales que podían atentar contra el orden establecido (Del Cura y Martínez, 2009. Del Cura, 2011). Ciencia y deficiencia, por ende, continuaron en el siglo XX como un binomio vigente. Además, las citadas carencias fueron atribuidas directamente a los sectores populares, vinculando así este problema a una cuestión social que se derivaba también desde el siglo anterior. En los discursos de diversas autoridades políticas, sanitarias e intelectuales, al igual que en las prácticas institucionales, la anormalidad continuó siendo asociada a la peligrosidad (De los Ríos y Rodríguez, 2007. Zapiola, 2010), requiriéndose para enfrentar dicho problema los aportes, perspectivas y opiniones de múltiples disciplinas, tales como la Medicina, la Psiquiatría, la Pedagogía y el Derecho.

¿Cuál fue la relación establecida entre el Estado chileno y la anormalidad? A grandes rasgos, evidenciamos una primera preocupación, más episódica y esporádica, que data del siglo XIX y que está referida en concreto hacia los sordo-mudos (Caiceo, 2010, p. 35). Pero, en el período aquí abordado, puede apreciarse tanto una relación fundada en la lógica de la prevención y control de conductas futuras, como en una acción dirigida a través de instituciones concretas y con proyección en el tiempo. Tal lógica implicará, a la larga, por parte de las autoridades estatales, la aceptación de una sociedad diversa y masiva cuyas necesidades requerirán una nueva organización y definición del ámbito educativo (Ponce de León, Rengifo y Serrano, 2012). No obstante, dicha inclusión en ningún caso eliminará las diferencias que igual se encargará de reproducir la denominada educación para anormales, categoría que desde la década de 1920 cobrará más fuerza y sentido en el plano discursivo e institucional, y que mantendrá su sentido hasta 1943, cuando se vuelva menos visible en las fuentes y se reoriente una de las instituciones claves en dicha materia: la Escuela Especial de Desarrollo. Siguiendo este razonamiento, la idea central que guía esta investigación busca comprobar que dicha educación para anormales no correspondería sólo a una lógica de intervención o protección social propia de un Estado Asistencialista, sino asimismo a la pervivencia de criterios preventivos y de control, más representativos de principios del siglo, que buscarían asegurar un orden social futuro, expresándose aún ideas propias de un modelo de Defensa Social. Por ello, no debe extrañar el énfasis en la infancia, ya que tal decisión obedecería a una razón práctica: estandarizar conductas y apariencias desde temprano para evitar peligros e inseguridades en el mañana. He ahí el papel preventivo y protector hacia los menores, pero también he ahí la construcción de un futuro ser social.

Las fuentes aquí utilizadas corresponden a escritos de época (artículos de revistas, documentación de archivo) provenientes tanto desde el ámbito médico, pedagógico y en menor medida legal. Si bien en ellos se combinan opiniones, diagnósticos, informaciones y referencias institucionales; en su totalidad permiten reconstruir un discurso común que enfatiza el cuidado 
terapéutico y educativo de los menores, aunque este mismo material muestra que cuando dichas ideas son llevadas al plano legislativo e institucional, se hace menos explícita la presencia de la medicina. He ahí un aspecto que, creemos, distingue a este proceso en Chile y que requiere, por supuesto, un desarrollo más amplio del que esbozaremos en las siguientes líneas.

\section{¿CUESTIÓN BIOLÓGICA O CUESTIÓN SOCIAL?}

Desde las últimas décadas del siglo anterior, los vínculos entre medicina y sociedad habían quedado configurados, ya fuese a través de la medicalización en tiempos de epidemias, como también por medio de la higiene social, cuya pretensión normalizadora y moralizadora terminó permeando clases, instituciones y espacios. La anormalidad representó a aquello que escapaba de la aceptación general de una sociedad y su cultura, vinculándose con las ideas de degeneración racial, física y mental que las elites y autoridades seguían restringiendo a una determinada clase social: los pobres. Diversas publicaciones a lo largo del período aquí abordado, siguieron reproduciendo e insistiendo en estas ideas, aunque no con la misma fuerza de antaño (León, 2003-2004), pero igualmente les dieron cabida en no pocas ocasiones con un respaldo "científico» que era avalado por algunos cuerpos editoriales. Los factores ambientales (geográficos, sociales, familiares) si bien eran puestos en relieve, no eran los únicos esgrimidos al momento de buscar explicaciones al aumento de la delincuencia o de la sensación de inseguridad que la prensa hacía notar como omnipresente en varias ciudades del país, ya que igualmente reaparecían las explicaciones deterministas que buscaban en la biología al «germen del mal» y en la medicina a la disciplina que podía encontrar una cura a esta enfermedad social que era el crimen. Para 1933, el doctor Luis Cubillos representará estas ideas cuando indique que:

las desviaciones físicas y psíquicas, grandes o pequeñas, [...] hacen del ser normal, empíricamente hablando, un ente antisocial, peligroso para la armonía colectiva, base única de bienestar y progreso. El estudio biológico de este hombre, que por sus antecedentes hereditarios, constitucionales, enfermedades y ambiente social es una entidad anormal, es el que realmente nos interesa (Cubillos, 1933, p. 47).

Tales diagnósticos no serán los únicos, pues aparte del citado galeno, otros contemporáneos arribarán a conclusiones similares (Brucher, 1944; Drapkin, 1943; Gajardo, 1944; Ramírez, 1935), después de examinar y clasificar a numerosos delincuentes. Incluso se lle- gará a afirmar que los que cometían delitos sexuales eran un "producto del instinto, o sea, del psiquismo elemental, siendo difícil que un sujeto inteligente caiga en este género de delitos» (Brucher, 1944, p. 103). La relación entre anormalidad, pobreza y criminalidad seguía vigente, pues eran todas muestras de lo patológico en su versión biológica, social y moral (Bilbao, 2011; Canguilhem, 1971; Foucault, 2006), exigiéndose al Estado, o a sus representantes, una mayor participación en la creación de políticas públicas e instituciones que resolvieran el problema, ya fuese con medidas represivas o preventivas. El objetivo, aunque no siempre explícito, apuntaba a vincular el bienestar de la nación con la salud física y moral del cuerpo social (Vallejo y Miranda, 2007, p. 17).

En esta perspectiva, se entiende que tomase sentido la preocupación estatal, primero de modo general, por la infancia y luego, de manera más específica, por la infancia anormal. La historiografía que ha estudiado el tema de la infancia en Chile, y que sigue de cerca los avances extranjeros sobre la materia, ha demostrado ampliamente que ya desde fines del siglo XIX la infancia no sólo se entendía como una etapa específica (entre el nacimiento y los 14 años), sino además había comenzado a recibir las miradas de la intelectualidad, las elites y las autoridades (Farías, 2003; Rojas, 2010). Aunque los principales avances legislativos e instituciones se hicieron más visibles desde la ley de instrucción primaria obligatoria (1920), todavía cobraban sentido en la discusión general algunas ideas elaboradas décadas antes. Una de ellas era la supuesta degeneración de la raza, motivo por el cual se atribuía a esta etapa formativa un rol fundamental en el futuro del individuo, ya que este era el momento para evitar anomalías e irregularidades que pudiesen causar daños al cuerpo social. De ahí que la biología se vinculara con la moral, pues se indicaba además que en la infancia se concentraban las inclinaciones positivas y negativas del ser humano, las cuales se conservaban aún en estado latente en la mente de los niños sin una clara delimitación (Foucault, 2007; Vandewalle, 2010).

La categoría infancia anormal, cuya presencia más sólida y clara puede datarse desde la década de 1890 en el viejo continente (Del Cura, 2011; Huertas, 2005; Muel, 1991), o desde principios del siglo XX, en el caso de algunos países latinoamericanos ( $\mathrm{Pa}$ dilla, 2012; Rossi, 2009; Vianna, 2007; De los Ríos y Rodríguez, 2007), terminó incluyendo dentro de sí a un heterogéneo conjunto de niños desvalidos, abandonados, vagos, delincuentes, deficientes mentales y deformes (Rossi, 2009). Frente a dicha diversidad, 
las respuestas buscaron más bien ser homogéneas, ya que se veía a todos ellos como un producto del mismo problema: la anormalidad. Ella era consecuencia del vicio (como el alcoholismo) y las costumbres depravadas (falta de moral y de higiene), pues los diagnósticos de médicos, juristas, jueces, publicistas e intelectuales terminaban vinculándola con la miseria, el hacinamiento, la ignorancia y el abandono familiar, entre otros males. Como este era el sustrato común de la anormalidad entre los menores, bastaba hacer referencia a él para catalogar a un niño de anormal, dejando inmediatamente así establecido su origen de clase.

En función de la proyección de muchas de estas ideas, es que puede comprenderse que dentro del proyecto de construcción de nación, por parte de los representantes del Estado Asistencialista, la infancia se considerase una etapa decisiva que requería intervención. Ya en la década de 1930 los diagnósticos y opiniones al respecto eran más explícitos. Cuando se hablaba de la vagancia infantil, se indicaba con total franqueza que el asunto "reviste una trascendencia social tal, [por lo] que su prevención debe ser una seria preocupación. Combatirla en todas sus formas, constituiría un gran paso hacia el mejoramiento físico y moral del hombre de mañana» (Mack E., 1930, p. 245). Otro balance posterior reiteraba ideas similares, indicándose que el niño "es un ser en formación. Sus actos no traducen una personalidad definitiva. Si su conducta es irregular, hay esperanza de modificarla mediante la formación de nuevos hábitos, y así, es su porvenir lo que más interesa a la sociedad» (Gajardo, 1935, p. 588).

Pero dicha intervención-protección no debía desvincularse de la prevención ni del control, pues, aunque niños, se les veía como seres potencialmente peligrosos que requerían de una tutela o una "pedagogía» que permitiera: normalizar sus conductas y comportamientos; regenerarlos -de ser posible-; utilizarlos e incorporarlos a la vida productiva; reafirmar sus sentimientos patrióticos y, en fin, corregirlos para la defensa y seguridad del cuerpo social. Valentín Letelier, un intelectual chileno preocupado por estas materias, en su obra Filosofía de la Educación (1927), lo dejaba bastante claro: "la pedagogía, que en el fondo es una higiene física, moral e intelectual respecto de los normales, se ha ennoblecido convirtiéndose en una ciencia médica y filantrópica a favor de los anormales» (Letelier, 1927, p. 595).

Pero intervenir la anormalidad implicaba legislar y crear instituciones encargadas de velar por el cum- plimiento de estas ideas, con el fin de evitar un «mal que se adivina» (Vianna, 2007), cruzada en la cual los saberes médicos, pedagógicos y judiciales debían estar unidos, para transformar la sociedad actual y asegurar la futura en aras de un mismo fin: regenerar la anormalidad hasta donde fuese posible o aminorar sus posibles efectos negativos. Lograrlo, era parte de la construcción de una sociedad más segura.

\section{TRES CARAS DE UNA MISMA MONEDA: INFANCIA DESVALIDA, DELINCUENTE Y DEFICIENTE}

Los espacios escolares que se gestaron dentro del período, e incluso antes (Serrano, Ponce de León y Rengifo, 2012), respondían a la atención que ameritaban grupos específicos de la población, en particular los menores anormales. Quienes tenían determinados rasgos físicos, sociales y morales, eran visualizados como seres especiales hacia los cuales debía existir una orientación igualmente especial (Caiceo, 2010). Como vimos, se partía del supuesto de que los estilos y formas de vida de estos niños, propios de los grupos populares, se manifestarían en inclinaciones viciosas que terminarían afectando el orden establecido. La prensa respaldaba estos prejuicios:

«Sucios, haraposos, pedigüeños, soeces en el hablar, [que] sugieren ideas bien poco halagadoras acerca de la sociedad a cuyo margen viven pero a la cual pertenecen en realidad» (La Nación, Santiago, 1928;

Citado en Rojas, 2007, p. 154).

Llama la atención la omisión a cualquier referencia sobre niños anormales de otra condición social. Las fuentes aquí revisadas, desde la prensa hasta los comentarios de los «especialistas» en esta materia, reproducen dicho silencio, lo cual no sólo hoy, sino también para el período analizado, terminaba por reafirmar para los contemporáneos la vinculación ya establecida entre pobreza y anormalidad. Más que buscar respuestas o explicaciones en las desigualdades que se generaban en los modelos políticos, sociales y económicos; se pensaba que el abandono, el delito o las causas físicas y biológicas estaban detrás del problema (Villalón, 1913; Flores, 1931; Sandoval, 1945). En este sentido la educación, y las escuelas que se crearan, debían apartar a los menores de la calle y evitar la reproducción de un mundo marcado por el desorden social y la desorganización familiar. Había, por ende, que establecer, parafraseando a Jacques Donzelot, un "complejo tutelar» que ayudara a condenar un modo de vida y a imponer otro, gracias al apoyo mutuo entre la medicina, la justicia y la pedagogía (Donzelot, 2008). 
La educación pensada para los niños anormales, al menos tal como aparece perfilada en algunas publicaciones como la Revista de Educación, terminaba igualmente relacionándose con el resto de la educación primaria, al enfatizar el desarrollo de las destrezas técnicas y manuales entre los menores para así generar conductas productivas, a la vez de sentimientos patrióticos y de obediencia a la autoridad. Por otra parte, lo que diferenciaba a esta educación, eran precisamente sus alumnos, los cuales requerían mayor atención y preocupación. Era preciso entender que necesitaban una enseñanza especial (específica) y especializada (que requería la conjunción de diferentes disciplinas y expertos para abordarla). En función de ello, se entiende que los diagnósticos relativos a su presencia dentro de la sociedad chilena, o más bien santiaguina en particular, terminaran entendiendo su necesidad, más aún cuando ya a fines de la década de 1920 estaba claro que dicha tarea debía estar en manos del Estado y sus representantes. ¿Cuáles fueron los centros de atención sobre esta materia? Básicamente tres: la infancia desvalida, la delincuente y la deficiente; desprendidas de las caracterizaciones y diagnósticos de los contemporáneos a la época en estudio.

Respecto de la infancia desvalida, la preocupación por los menores abandonados y proclives a caer en los vicios de la calle, por su condición vulnerable, estuvo presente desde el siglo XIX, pero concentrada en los particulares y algunos miembros de la Iglesia Católica. La percepción del aumento de estos menores en las ciudades, como asimismo un mayor interés en la infancia como etapa vital clave para conductas futuras, llevaron a que se definiera progresivamente una mayor preocupación estatal, la que con vacíos y ciertas ambigüedades (Letelier, 1918) se tradujo en un primer Congreso de Protección a la Infancia desvalida (1912) y en una ley sobre la materia (1912), que fue corregida y complementada por una Ley de Menores en 1928. Los estudios sobre el particular (Farías, 2003; Rojas, 2007 y 2010), han hecho notar que detrás de estas medidas no había una real «consistencia teórica» y que no hubo gran "debate sobre la necesidad de satisfacer ciertas necesidades materiales y afectivas» (Rojas, 2007, p. 130 y 159). Lo que no debe extrañar, pues el discurso de protección a la infancia estuvo atravesado con frecuencia por el de la defensa social, dando en ocasiones resultados paradójicos, contradictorios o complementarios con los criterios asistencialistas.
Los trabajos que han investigado esta idea de la «salvación de los niños» (Platt, 1982), han expuesto cómo detrás de la legislación y la institucionalidad existía el criterio de que el menor sólo tomaba decisiones erradas, requiriendo por ello de una permanente tutela. Gran parte de los comentarios de la época sobre el tema, terminaron combinando en sus juicios y acciones criterios de protección y vigilancia que más que contradictorios se volvían complementarios. Por ello, es comprensible que en esta lógica el menor fuese concebido como un sujeto de deberes y no de derechos (Farías, 2003), a pesar de que existiera una retórica que hablaba de los «derechos del niño», como bien lo hicieran los escritos del juez de Santiago Samuel Gajardo durante la década de 1940 (Rojas, 2007, p. 142).

En la infancia delincuente, o peligrosa, persistía la ambigüedad del discurso, dado que se buscaba prevenir y controlar conductas futuras mediante la formación de una ética del trabajo industrial, una disciplina militarizada y diversas rutinas que enfatizaban el control del cuerpo (educación física), la mente (instrucción de primeras letras y de moral cívica) y el espíritu (enseñanza religiosa). La institución que encarnó dichos ideales fue la Escuela Correccional de Menores (León, 2003), creada en Santiago en 1896 y que encontró réplicas en otras ciudades de Chile, aunque con diferente proyección en el tiempo: Talcahuano (1896), Valparaíso (1900), Concepción (1906). Detrás de dicho proyecto estaba el cuestionamiento al ambiente familiar y social, pues como proyección del siglo XIX se pensaba que la familia debía ser el «espacio educativo que iniciara a los nuevos ciudadanos en las virtudes públicas del bien común como en las privadas del orden y el trabajo» (Ponce de León, Rengifo, Serrano, 2006, pp. 43-44). Para nuestro período, ya estaba claro que este rol debía ser competencia del Estado y no de los particulares. En función de un cambio en algunos criterios, la Escuela correccional en 1913 pasó a llamarse Escuela de Reforma de Santiago, mientras que los cambios más significativos tuvieron lugar desde 1924 como veremos. Dichos cambios buscaban darle al establecimiento un carácter más pedagógico y menos carcelario, pero pese a los cambios de nombres y a reformas puntuales, dicha institucionalidad estaba transitando a un modelo más proteccionista y asistencial, aunque sin olvidar las funciones preventivas y de vigilancia (Ricci, 1930; Roccuart, 1932).

La infancia deficiente, se concentraba en menores con retardo mental, y había tomado visibilidad en algunas publicaciones educacionales desde mediados 
del siglo XIX, aunque no de forma continua (León, 2014). Se entendía como un ámbito que debía ser intervenido, corregido, fiscalizado y prevenido. Aunque en el discurso oficial se asumía la diversidad, ello no se traducía necesariamente en una inclusión, pues se establecía de antemano, al igual que en las correccionales, el sentido práctico o utilitario que debía tener dicha educación (Schweitzer, 1938), descartándose cualquier otro tipo de enseñanza a priori. Ese fue el espíritu que dio forma y fondo a la Escuela Especial de Desarrollo a partir de 1929, que combinaba la educación, la asistencia, la prevención y el control de los menores allí internos. Lo que se buscaba resolver era el problema de una infancia destutelada, fuera de las tutelas familiares, paternales, religiosas, sociales y de la ciencia. Por ende, la educación pensada no sólo para deficientes, sino también para delincuentes y desvalidos, buscaba restablecer dichas tutelas, para sanar los posibles males sociales que pudiesen surgir (Sandoval, 1937).

En suma, la categoría infancia anormal hasta aquí revisada, y que hemos visualizado sumariamente en las caracterizaciones abordadas, no sólo supone considerar las relaciones sociales que hacen posible la aparición de la infancia moderna a través de redes institucionales como la familia y la escuela, sino también ayuda a comprender cómo el campo de la infancia se transforma en un objeto de conocimiento y de intervención social. Las tres infancias aquí explicadas componen y definen una anormalidad construida a partir de criterios preventivos, asistenciales y de control, respaldados por saberes médicos y pedagógicos que no sólo enfatizan las ideas de prevención y tratamiento, sino además exigen la identificación previa de estos niños y su reclusión en establecimientos. El objetivo, como hemos apuntado, era evitar que estos menores se convirtieran en seres inútiles, desadaptados e improductivos, en ciudadanos extrasociales (vagabundos, mendigos) o asociales (criminales peligrosos) (Del Cura, 2011; Fernández, 2012).

\section{TRANSFORMACIONES EN LA LEGISLACIÓN Y LA INS- TITUCIONALIDAD}

Para mediados de la década de 1920 se comienzan a evidenciar cambios en los criterios para abordar el tema de la infancia anormal. Ya en 1924 el entonces ministro de Instrucción, Luis Salas Romo, solicitaba al Director de Instrucción Primaria que estudiase la formación de internados-talleres para acoger a los menores desvalidos con un fin claro, que se transformaran en "factores de utilidad social para sacarlos de una vida miserable que los dejaría como seres degenerados o inadaptados» (El Diario Ilustrado, Santiago, 30 de julio de 1924). La fecha es significativa, dado que ese mismo año se propuso la reorganización de la Escuela de Reforma de Santiago, que se transformó en la Escuela de Educación Preventiva, ahora bajo la dependencia del Ministerio de Previsión Social. El ideal preventivo que afectaba al nombre del establecimiento buscaba dar a entender un nuevo sentido de asilo para los niños «en situación de delinquir, previniendo que se transformasen en pequeños criminales» (El Diario Ilustrado, Santiago, 30 de octubre de 1924). Tal criterio también se extendió a otras instituciones, lo que quedó claro al año siguiente al crearse, con fines más amplios que atender a la infancia desvalida y delincuente, la Sección especial de establecimientos penales y preventivos contra la delincuencia (Decreto Ley 491, en Diario Oficial, Santiago, 26 de agosto de 1925).

La ley de menores de 1928 dispuso una nueva estructura institucional, que junto con proteger y reforzar los derechos de los menores, incluyó ideas propias de la defensa social. De hecho, ya en el proyecto de ley presentado al Ejecutivo un año antes (4 de agosto de 1927), se indicaba al hablar de los delitos en los niños, que debe "aplicárseles un tratamiento médico y pedagógico, tratando de transformarlo en un elemento útil, y a la vez defender a la sociedad de él, conforme a su grado de temibilidad" (Citado por Rojas, 2010, p. 385). Esta ley igualmente se encargó de crear instituciones como la Dirección General de Protección de Menores, a cargo del Ministerio de Justicia, entre cuyos fines estaba el «atender al cuidado personal, educación moral, intelectual y profesional de los menores abandonados, delincuentes o en peligro moral o material» (Diario Oficial, Santiago, 9 de enero de 1929). Asimismo, se crearon Casas de Menores en Santiago (1929) y Valparaíso (1930). Recogiendo algunas de estas ideas, en 1934 se planteó la organización de un Consejo de Defensa del Niño Abandonado, que encontró finalmente respaldo gubernamental, como bien lo indicaba la prensa cuando se decía que: «Chile requiere para su desarrollo, que comienza ahora a tomar tanta importancia por el fortalecimiento de sus industrias propias, de una población moralmente firme y físicamente resistente» (La Nación, Santiago, 9 de junio de 1934). El presidente Arturo Alessandri, respaldando dichas ideas y el Consejo, señalaba que no era posible «abandonar al niño desvalido, que será parte integrante de la patria de mañana» (El Mercurio, Santiago, 9 de junio de 1934). 
Si bien la ley de menores buscó mantener criterios de cuidado sanitario general para los menores, el papel de los médicos en esta legislación no quedó claramente establecido, pues en la práctica las organizaciones de protección aquí mencionadas dependieron administrativamente del Ministerio de Justicia. Sólo en 1942 se introdujeron cambios drásticos quedando todas estas organizaciones bajo el Ministerio de Salubridad (Ley 7200 en Diario Oficial, Santiago, 18 de julio de 1942). Por un decreto del 7 de octubre de ese año, se refundieron asimismo varias instituciones en la Dirección General de Protección a la Infancia y Adolescencia (DGPIA), en cuya reglamentación se empleó por primera vez un concepto que desplazaba a los ya conocidos: "Se entenderá que un menor se encuentra en situación irregular, cuando su adaptación social sufriere alteraciones, se encuentre moral o materialmente abandonado o en peligro de estarlo, o hubiere delinquido, cualquiera sea su estado civil» (Reproducido en Diario Oficial, Santiago, 31 de octubre de 1942). Desde esta fecha en adelante, constatamos un menor uso en la documentación de la categoría anormalidad. La nueva Dirección no abandonó su carácter preventivo, pues si bien dependió del M. de Salubridad, sus funciones requirieron la coordinación con otras instituciones públicas como el Ministerio de Educación, del Trabajo, del Interior (a través de la Dirección General de Auxilio Social y la Dirección General de Informaciones y Acción Cultural) y las cajas de previsión social, definiendo así un panorama que duraría hasta 1952 cuando desaparezca la DGPIA.

\section{PEDAGOGÍA Y REGENERACIÓN}

Durante la década de 1930 se hicieron evidentes, por parte de la prensa y del discurso político, una serie de temores derivados de la crisis de 1929. Se vio en el Estado a un garante del orden social, pero también a un ente preventivo que debía velar por alejar los temores, reales o imaginarios, de la población. En dicha línea se sustentó una búsqueda proyectiva de seguridad, haciéndose necesario para ello la intervención de la pobreza, la infancia popular y, dentro de ella, la anormalidad. Enfrentar este último aspecto implicaba ayudar a superar, por ejemplo, las impredecibles conductas que se atribuían a los pobres y más aún a quienes tenían un retardo mental. Una política preventiva y de control hacia los menores, concebidos como los futuros ciudadanos, no sólo tranquilizaba en lo inmediato, sino asimismo permitía planificar y modelar las actitudes del mañana.
Institucionalmente, como se adelantó, se crearon en Santiago una Casa de Menores y una Escuela Especial de Desarrollo (Boletín de Escuelas Experimentales, no 1, 1929-1930.) orientadas a dar forma y contenido a las ideas aquí expuestas:

El fin primordial en la enseñanza de los subnormales (término que aparece utilizado por primera vez en lo que hasta el momento hemos investigado), es producir individuos respetuosos de la ley, sociales y capaces de levantar su propio peso en la sociedad donde viven. De aquí se deduce la labor fundamental del profesor que es la educación del carácter por la inculcación de hábitos precisos de buena conducta y de buen ciudadano (Flores, 1931, p. 863).

En dichos espacios se buscó complementar lo biológico y lo social, la medicina y la pedagogía, la cual en dicho escenario podía tomar un papel regenerador, interviniendo una naturaleza defectuosa para encauzarla hacia "hábitos deseables» (Latorre, 1944, p. 18). Ambas disciplinas debían ayudar a definir, delimitar y clasificar a los menores anormales (niños y niñas) en aras de ir desentrañando las complejidades de este nuevo saber (la anormalidad) y de entregar criterios para una también nueva práctica pedagógica (la educación de anormales), que considerase tanto las denominadas clases diferenciales como la tutela permanente a través de secciones de internado o transformando a las escuelas en internados.

Es aquí donde cobra un papel relevante el desarrollo y perfeccionamiento de los test, de mediciones de coeficiente intelectual y de diagnósticos que permitieran realizar una nosología (clasificación de anormales), indicadora de los grados de anormalidad, de la posible reinserción social y de la institución específica que debía educar o reeducar al menor. Por supuesto, de ser negativas estas pruebas, el niño era catalogado como anormal, concentrándose la preocupación ya no en incrementar su intelecto, sino en evitar su desadaptación social. Ello, porque dichos anormales eran presentados como incapaces, improductivos e imposibilitados para cualquier trabajo intelectual. Así se entiende la minuciosidad de trabajos como el del doctor Hugo Lea-Plaza en la sección de observación de la Casa de Menores, realizando "una individualización dactiloscópica, un informe social, un diagnóstico psicológico y exámenes pedagógicos, médicos y antropológicos» (Lea-Plaza, 1929, p. 11). También se comprende la implementación en las escuelas primarias de métodos de diagnóstico temprano, como los de Ovide Decroly y María Montessori, elaborándose fichas con antecedentes familiares, mediciones del 
coeficiente intelectual y exámenes de nutrición; que ayudaban a evaluar las condiciones de incorporación de estos niños a las escuelas (Torres, 1933, p. 7).

En el caso de los alumnos con mayor retraso mental, como los idiotas e idiotas profundos -unas de las tantas clasificaciones usadas entonces-, se decía que los exámenes aplicados a ellos, a nivel sensorial y motriz, podían tardar meses, incluso años en dar resultados, pues por lo general estos menores no mostraban avances y, en el peor de los casos, no respondían a los estímulos (Flores, 1931). Mejorar la expresión lingüística, la coordinación motora, la ubicación espacial y las habilidades motrices y sensoriales; fueron las metas de los maestros para llevar adelante un proceso pedagógico y terapéutico.

Las escuelas especiales se pensaron para aquellos casos de anormalidad cuya complejidad ameritara tratamiento psiquiátrico, médico y farmacológico de carácter constante para incentivar el aprendizaje, así se evitaban daños a los niños normales y el retroceso en los aprendizajes y socialización de los anormales. Ese fue el sentido de la Escuela Especial de Desarrollo como su nombre bien lo indica. Aquí la segregación fue vista como una medida preventiva, pues de registrarse avances en un niño anormal, se buscaba su reinserción en una escuela normal. Esto obedecía a que se pensaba que los niveles de presión e instrucción de la educación formal, podían ser tolerados sólo por niños medianamente inteligentes (Flores, 1931; Latorre, 1943; Salas, 1931).

Había voces que explicitaban la complicada labor de educar a estos niños en el mismo espacio que a otros menores normales, no debiendo confundirse ni conceptos ni criterios. Enriqueta Laferriere, a comienzos de los años treinta, criticaba las propuestas de instruir con rapidez a los niños anormales e insertarlos en los estudios primarios, pues, en su opinión, las denominadas clases diferenciales: "se adecuaban para que los atrasados pedagógicos no perdieran contacto con el resto de sus compañeros y no salieran de la escuela, adaptándose aulas dentro de los colegios para tales fines y dejando a los recreos y a las clases de educación física como las instancias de reinserción y socialización». Pero otra cosa muy distinta era la anormalidad, que visualizaba a través de tres categorías, la de los débiles mentales (quienes podían ganarse la vida, pero eran incapaces de gobernarse a sí mismo y competir con normales), los imbéciles (por defecto de nacimiento o de la primera infancia, incapaces de ganarse la vida, pero podían guardarse a sí mismos), y los idiotas (incapaces de protegerse a sí mismos). Por conclusión las clases diferenciales no debían recibir "a ninguno de estos niños, los que necesitan escuelas especiales y el cuidado de médicos especialistas. Las clases diferenciales son para los retardados pedagógicos o falsos anormales» (Laferriere, 1933, p. 49).

Otro punto que requería la intervención pedagógica era el del ausentismo escolar, pues la anormalidad era vista como un determinante en la incapacidad para lograr avances en los menores. Si bien tal problema no se presentaba sólo en estos niños, pues desde el siglo XIX era una reflexión recurrente (Monsalve, 1998), aquí se agregaba el hecho de que a nivel familiar dicha anormalidad era visualizada como una carga involuntaria en la que niños y adolescentes pasaban a ser dependientes y un costo extra para la economía familiar. El profesor Claudio Salas, desde las columnas de la Revista de Educación, indicaba que a pesar de que las autoridades educacionales buscaban obligar a los padres a mandar a sus hijos a las escuelas, mediante la aplicación de sanciones, multas e intervención policial inclusive; era preciso atender a la raíz del problema y que era:

La herencia sifilítica, alcohólica, neuropática, tuberculosa, explica etiológicamente la producción de perturbaciones nerviosas del carácter mental de que están atacados los niños indisciplinados y de frecuentación irregular [...] Los niños que están atacados presentan una psicología anormal, de la que la no-frecuencia escolar constituye su primera manifestación de la indisciplina y de acción anti social. Ellos resbalan, además, más o menos, rápidamente hacia el vicio y la delincuencia (Salas, 1931, p. 52).

No obstante el protagonismo que se le asignó a la medicina en el tema, en el plano legislativo como institucional aparece con menor presencia, o al menos así lo registran las fuentes hasta aquí revisadas. De hecho, si bien no puede negarse la presencia de médicos en los establecimientos, las publicaciones de la época muestran una escasa presencia de doctores escribiendo $u$ opinando sobre el particular. Abundan los balances pedagógicos en la Revista de Educación, pero las voces de los galenos, y de los psiquiatras específicamente, no son explícitas al respecto, con la excepción del doctor Gustavo Vila quien en 1935, al evaluar el papel del pedagogo en estos temas, hacía notar su incapacidad para asistir a un deficiente mental al carecer de preparación suficiente en el ámbito de la psicología. No era posible, a su entender, cumplir los roles de médico y maestro a la vez al desconocerse las psico-patologías y la psiquiatría infantil (Vila, 1935, p. 39). Para médicos y psiquiatras era difícil concebir 
la rehabilitación de un anormal, entendiendo a la educación como una mera instrucción de modales, pues sólo se podía reacondicionar al individuo para obedecer lecciones en aras de mantener el orden social. Para los pedagogos, en cambio, era posible la rehabilitación basada en un aprendizaje integral.

Así cobraba sentido una Escuela Especial de Desarrollo que, con carácter mixto, fue concebida gracias a la asesoría del estadounidense Lloyd Yepsen, para quien «El niño tardo [debía ser] colocado en una clase especial a fin de que no vaya demasiado ligero [...] el propósito ideal sería que cada niño al ingresar por primera vez a la escuela pública, fuera sometido a un estudio completo que comprendiera, no sólo el examen psicológico y mental, la manera cómo reacciona, el sistema emocional, etc. sino también el examen de un médico y de otros especialistas agregados al servicio escolar» (El Mercurio, Santiago, 8 de julio de 1929). A los pocos años de funcionamiento, en un predio rural de La Cisterna, se hizo evidente la necesidad de anexar una sección de internado, lo cual se concretó en 1933 (Decreto 4259, Santiago, 31 de octubre de 1933, en ARNAD. Vol. 6124. José Flores. "Proyecto de fundación de un internado anexo a la Escuela Especial de Desarrollo, Santiago, 1933, en $A R N A D$. Vol. 6125.). Tal iniciativa deseaba responder a un interés concreto: "atender [...] mediante un criterio psicopedagógico y médico a estos seres de escaso nivel intelectual, a fin de contrarrestar, hasta donde se pueda, el efecto terrible de sus taras, salvaguardando así los actuales y futuros intereses económicos, sociales y morales de la nación" (Claudio Salas. «Solicita aprobación proyecto Internado Anexo a la Escuela Especial de Desarrollo, Santiago, 18 de agosto de 1933, en ARNAD. Expediente no 10306).

Sólo para 1943 se produciría otro gran cambio, pues de acuerdo con una reseña escrita sobre el establecimiento: "La Escuela sintió la imperiosa necesidad de ser Internado, como único medio de posibilitar la acción integral del niño retardado mental y de capacitarlo, sin la mediación de estímulos perturbadores, para aquella eficiencia social compatible con sus disposiciones mentales» (Revista de Educación, no 26, Santiago, 1944, p. 408).

Para entonces su director, Gonzalo Latorre, plantearía lo que debían ser las líneas a seguir en la materia, dada:

la enorme legión de los insuficientes psíquicos que día a día incrementan el ya enorme ejército de los tarados sociales, ejército de los incapaces de gober- narse a sí mismo, de los sugestionables y de los casi anormales, plantea a nuestra nacionalidad un grave problema social, cuya solución tiene sólo dos caminos: a) el asistencial y b) el educativo. El primer camino no elimina las causas que originan el mal y cuesta permanentemente considerables sumas de dinero; el segundo lo impone el sentido de solidaridad social del presente y la esencia de los postulados democráticos que señalan a cada individuo un lugar en su servicio a la comunidad social (Latorre, 1943, p. 20).

Si bien nuestro interés no ha sido reconstruir institucionalmente la trayectoria de esta Escuela-Internado, las expresiones de sus directivos nos ayudan a reforzar no sólo el discurso preventivo y controlador hacia la infancia anormal que aquí se hace explícito, sino asimismo a vincularlo con búsquedas más globales del período como el mejoramiento de la raza y, en concreto, con las políticas eugenésicas que terminaron reforzando la relación establecida entre salud y educación (Cabrera, 2014. Cid, 2009). Se aprecia asimismo una mayor vinculación entre anormalidad y deficiencia mental que a comienzos del siglo XX, o al menos así se desprende del material revisado, lo que muestra la historicidad de la categoría anormalidad, aunque todavía no se evidencia su extensión a otros grupos sociales. Esa, quizás, será la característica del período siguiente.

\section{CONCLUSIONES}

A lo largo del período revisado en esta investigación, es posible apreciar cómo la relación entre el Estado y la anormalidad presentó cambios respecto de comienzos del siglo. Con más claridad desde la década de 1920, las autoridades políticas, en conjunto con los saberes médicos y pedagógicos, buscaron incluir a la excluida anormalidad infantil dentro de un proyecto de desarrollo nacional, industrial y de formación de ciudadanos. A pesar de esta nueva lógica, la segregación como tal no desapareció, pues persistieron criterios que subsumían las diferencias de género, que vinculaban dicha anormalidad a una sola clase (los pobres) y que se concentraban sólo en las deficiencias físicas y mentales más evidentes y que pudiesen provocar una futura peligrosidad; no diciéndose nada de ciegos, sordos y mudos. En tal lógica, junto a la protección y asistencia hacia los menores, coexistía un ánimo de prevención, vigilancia y control. De ahí que en el proyecto estatal chileno se priorizara, a diferencia de lo que concluyen estudios sobre esta materia en otras realidades históricas y geográficas, a la pedagogía antes que a la medicina al momento de tomar 
decisiones sobre la práctica o funcionamiento de las instituciones reformadas o surgidas después de la ley de menores de 1928. Tal situación no es extraña si se piensa que los diagnósticos de la primera prometían regenerar, o reeducar de ser posible, a estos menores, a diferencia de la segunda, que tendían a ser más deterministas y poco esperanzadores en sus balances. Por ello, se comprende que con el respaldo de la pedagogía, el Estado orientara la anormalidad infantil hacia un fin productivo. Así se aseguraba el mañana y se redefinía una relación que hasta entonces no había sido concebida bajo estos parámetros.

Desde que la ley de instrucción primaria obligatoria (1920) buscó como ideal una educación tripartita: física, moral e intelectual; los esfuerzos se concentraron en ir perfeccionando aquellas áreas educativas y de protección al menor que el Estado Asistencialista perfiló y consolidó. La anormalidad infantil no sólo se definió, clasificó y buscó ser intervenida y regenerada

\section{NOTA}

* Investigación derivada del Proyecto FONDECYT no 1140777.

\section{FUENTES PRIMARIAS}

Archivos: Archivo Nacional de la Administración (ARNAD). Vols. 3164, 6124, 6125; Expediente no 10306.

Ley № 3654 sobre Instrucción Primaria Obligatoria (1920), Santiago, Imprenta Lagunas \& Co.

\section{BIBLIOGRAFÍA}

Álvarez Uría, Fernando (1991), "Los niños anormales. Constitución del campo de la infancia deficiente y delincuente". En: Arqueología de la escuela, Madrid, Las ediciones de La Piqueta, pp. 209-234.

Álvarez Uría, Fernando (1996), “La configuración del campo de la infancia anormal. De la genealogía foucaultiana y de su aplicación a las instituciones de educación especial”. En: Barry M. Franklin (comp.), Interpretación de la discapacidad. Teoría e historia de la educación especial, Barcelona Ediciones Pomares \& Corredor, pp. 90-124.

Bilbao, Alejandro (2011), Las creaciones freudianas de lo patológico. Sujeto, causa y representación. Santiago, Universidad Academia de Humanismo Cristiano.

Brucher Encina, Hernán (1944), Estudio de la personalidad de los delincuentes chilenos. Santiago, Editorial La Salle. a través de una nueva legislación e institucionalidad, sino también comenzó a ser comprendida desde una renovada terminología; apareciendo por primera vez la expresión «menores en situación irregular» (1942); y desde una reformulada institución que entendió la necesidad de una tutela permanente: la Escuela Especial de Desarrollo y su transformación en Internado. A pesar de no contar con mayores visiones críticas ni internas de dicha institucionalidad, pues las fuentes se muestran más esquivas a este respecto, es posible desprender de los discursos que legitimaban este panorama, como aún se seguía proyectando a los grupos populares la probabilidad de un eventual desorden futuro y que la anormalidad física y mental reproducía la de carácter moral. Tales ideas fueron más bien propias de un modelo estatal de defensa social que no desapareció por completo, sino que se mantuvo de la mano con una tendencia benefactora, industrialista y de mejoramiento de la raza, que también cobró protagonismo durante las décadas de 1930 y 1940.

Prensa: El Diario Ilustrado, El Diario Oficial, El Mercurio, La Nación.

Caiceo Escudero, Jaime (2010), “Esbozo de la educación especial en Chile: 1850-1980", Revista Educación y Pedagogía. Facultad de Educación, Universidad de Antioquia, Medellín, 22 (57), mayo-agosto, pp. 31-49.

Canguilhem, Georges (1971), Lo normal y lo patológico. Buenos Aires, Siglo XXI Editores.

Cid, Gabriel (2009), "Médicos, abogados y eugenesia negativa en Chile, 1933-1941", Anales de Historia de la Medicina, 19, pp. 35-46.

Correa, Sofía et al. (2001), Historia del siglo XX chileno. Santiago, Editorial Sudamericana.

Cubillos, Luis (1933), “Criminología y antropología médica penitenciaria", Revista de Estudios Penitenciarios, 4, pp. 42-47. 
Del Castillo, Alberto (2005), “Médicos y pedagogos frente a la degeneración racial: La niñez en la ciudad de México, 18761911". En: Claudia Agostoni y Elisa Speckman (eds.), De normas y transgresiones. Enfermedad y crimen en América Latina, 1850-1950, México, UNAM, pp. 83-107.

Del Cura, Mercedes y José Martínez Pérez (2009), “Entre la amenaza y la integración: sobre el discurso acerca de la deficiencia mental en España (1900-1936)". En: Marisa Miranda y Álvaro Girón (coords.), Cuerpo, biopolítica y control social. América Latina y Europa en los siglos XIX y XX., Buenos Aires, Siglo XXI Editores, pp. 95-114.

Del Cura, Mercedes (2011), Medicina y Pedagogía: la construcción de la categoría "infancia anormal" en España. Madrid, CSIC.

De los Ríos, Alexander Yarza y Lorena M. Rodríguez (2007), Educación y pedagogía de la infancia anormal, 1870-1940. Bogotá, Cooperativa Editorial Magisterio.

Dirección General de Educación Primaria (1929-1930), Boletín de Escuelas Experimentales, 1, Santiago.

Donzelot, Jacques (2008), La policía de las familias. Buenos Aires, Ediciones Nueva Visión.

Drapkin, Israel (1943), “Observaciones acerca de un centenar de delincuentes chilenos examinados en el Instituto de Criminología de Chile", Boletín de la Dirección General de Prisiones, 63-67, Santiago.

Farías, Ana María (2003), "El difícil camino hacia la construcción del niño como sujeto de derechos", Revista de Derechos del Niño, 2, pp. 187-224.

Fernández Labbé, Marcos (2012), “Asociales: raza, exclusión y anormalidad en la construcción estatal chilena, 1920-1960", en Revista de Historia Social y de las Mentalidades. Vol. 16, no 2, USACH, Santiago, pp. 167-194.

Flores, José (1931), "Reporte trimestral sobre la pedagogía de los niños anormales", en Revista de Educación, 25, Santiago, pp. 863-901.

Foucault, Michel (2006), La vida de los hombres infames. Buenos Aires, Ediciones Altamira.

Foucault, Michel (2007), Los anormales. México, F.C.E.

Gajardo, Samuel (1935), "La readaptación social del niño vago", Boletín de la Dirección General de Protección de Menores, Santiago, enero, 6, pp. 587-591.

Gajardo, Samuel (1942), Ensayo sobre el hombre antisocial. Santiago, Imprenta Cervantes.

Huertas, Rafael (1998), Clasificar y educar. Historia natural y social de la deficiencia mental. CSIC, Madrid.

Huertas, Rafael (2005), “La medicalización de la delincuencia infantil en la España del primer tercio del siglo XX”. En: Marisa Mirada y Gustavo Vallejos (comps.), Darwinismo social y eugenesia en el mundo latino, Buenos Aires, Siglo XXI Editores, pp. 375-400.

Illanes, María Angélica (2007), Cuerpo y sangre de la política. La construcción histórica de las Visitadoras Sociales (18871940). Santiago, LOM Ediciones.

"La creación del Primer Internado para Niños Deficientes Mentales" (1944), en Revista de Educación, 26, Santiago, pp. 405-409.

Laferriere, Enriqueta (1933), "Las clases diferenciales", Revista de Educación, Santiago, 24, pp. 38-43.

Latorre, Gonzalo (1943), "Problemas educativos de los retardados mentales: Bases para la organización de una enseñanza especial del niño deficiente mental", Revista de Educación, Santiago, 16, pp. 17-22.

Lea-Plaza, Hugo (1929), "La sección de observación de la Casa de Menores de Santiago", Boletín de la Dirección General de Protección de Menores, Santiago, julio, 1, pp. 11-15.

León León, Marco Antonio (2003-2004), “Los dilemas de una sociedad cambiante: Criminología, criminalidad y justicia en Chile contemporáneo (1911-1965)", Revista Chilena de Historia del Derecho, 19, pp. 223-277.

León León, Marco Antonio (2003), Encierro y corrección. La configuración de un sistema de prisiones en Chile (1800-1911), Tomo III. Santiago, Universidad Central de Chile.

León León, Marco Antonio (2015), “Educación, regeneración y punición para la 'infancia anormal': Las escuelas correccionales de Talcahuano y Concepción, Chile (1896-1915)". En: Jorge Alberto Trujillo Bretón (coord.), Voces y memorias del olvido. Historia, marginalidad y delito en América Latina, siglos XIX y XX, México, Universidad de Guadalajara, pp. 351-400.

Letelier González, Hipólito (1918), La protección de la infancia. Santiago, Imprenta S.B.

Letelier, Valentín (1927), Filosofía de la Educación. Cabaut y Cía. Buenos Aires, Editores.

Mack E., Olga (1930), "La vagancia de los niños", Boletín de la Dirección General de Protección de Menores, Santiago, enero, 3, pp. 245-262.

Monsalve, Mario (ed.) (1998), "I el silencio comenzó a reinar". Documentos para la historia de la instrucción primaria, 1840-1920. Santiago, DIBAM.

Muel, Francine (1991), "La escuela obligatoria y la invención de la infancia anormal". En: Robert Castel et al. Espacios de poder. Madrid, Las Ediciones de La Piqueta, pp. 123-142.

Padilla Arroyo, Antonio (Coord.) (2012), Arquetipos, memorias y narrativas en el espejo. Infancia anormal y educación especial en los siglos XIX y XX. México D.F., Universidad Autónoma del Estado de Morales. 
Ponce de León, Macarena; Francisca Rengifo y Sol Serrano (2006), “La 'pequeña república'. La familia en la formación del Estado Nacional, 1859-1929”. En: J. Samuel Valenzuela; Eugenio Tironi y Timothy Scully, c.s.c. (eds.) El eslabón perdido. Familia, modernización y bienestar en Chile. Santiago, Taurus, pp. 43-96.

Ramírez Uribe, Crisólogo (1935), La colonia penal y la readaptación del delincuente. Escuela de Derecho. Santiago, Universidad de Chile.

Ricci Ferrari, Armando (1930), La delincuencia infantil y los tribunales para menores. Estudio comparado de la Ley № 4447. Santiago, Imprenta del Ministerio de Guerra.

Roccuart Hidalgo, J. Félix (1932), La delincuencia infantil y los reformatorios de niños. Santiago, Imprenta La Tarde.

Rojas Flores, Jorge (2007), “Los derechos del niño en Chile: una aproximación histórica, 1910-1930", revista Historia, vol. I (40), pp. 129-164.

Rojas Flores, Jorge (2010), Historia de la infancia en el Chile republicano, 1810-1910. Santiago, JUNJI.

Rossi, Gustavo Pablo (2009), “El clima de época en la 'anormalidad' infantil: deficientes-abandonados-vagos-delincuentes, 1880-1930", Temas de Historia de la Psiquiatría Argentina, 28, pp. 17-26.

Salas, Claudio (1931), "El problema de la no-frecuentación escolar. Las causas de anomalías del carácter y mentales", Revista de Educación, 28, pp. 51-53.

Salazar, Gabriel y Julio Pinto (1999), Historia contemporánea de Chile, Tomo I. Santiago, LOM Ediciones.

Sandoval Carrasco, Juan (1937), "Orientación profesional con el niño retardado mental", Revista de Educación, 82, pp. 5-18.

Sandoval Carrasco, Juan (1945), El niño retardado mental. Santiago de Chile, Publicaciones de la Escuela Especial de Desarrollo.
Schweitzer, Miguel (1938), "Síntesis de la legislación chilena de menores", Revista de Ciencias Penales, julio-agosto, 21, pp. 310-316.

Serrano, Sol; Macarena Ponce de León y Francisca Rengifo (eds.) (2012), Historia de la educación en Chile (1810-2010). Tomo II. La educación nacional (1880-1930). Santiago, Taurus.

Speckman, Elisa (2005), "Infancia es destino. Menores delincuentes en la ciudad de México (1884-1910)". En: Claudia Agostoni y Elisa Speckman (eds.) De normas y transgresiones. Enfermedad y crimen en América Latina, 1850-1950, México, UNAM, pp. 225-253.

Torres, Luis (1933), "El Doctor Decroly: Su personalidad, sus principios y su obra", Revista de Educación, 42, pp. 7-30.

Vallejo, Gustavo y Marisa Miranda (comps.) (2007), Políticas del cuerpo. Estrategias modernas de normalización del individuo y la sociedad. Buenos Aires, Siglo XXI Editores.

Vandewalle, Bernard (2010), "La escuela y los niños 'anormales'. El análisis de Michel Foucault”, Revista Educación y Pedagogía. Facultad de Educación, Universidad de Antioquia, Medellín, mayo-agosto, 22 (57), pp. 203-215.

Vianna, Adriana (2007), El mal que se adivina. Policía y minoridad en Río de Janeiro, 1910-1920. Buenos Aires, Ad-Hoc Ediciones.

Vila, Gustavo (1935), "Psicología escolar", Revista de Psiquiatría y disciplinas conexas, $U$. de Chile, 1, pp. 25-39.

Villalón, Elena (1913), “Contribución al sistema educativo de los niños anormales", en ARNAD. Vol. 3164

Yáñez, Juan Carlos (2008), La intervención social en Chile, 19071932. Santiago, RIL Editores.

Zapiola, María Carolina (2010), "Espacio urbano, delito y 'minoridad': aproximaciones positivistas en el Buenos Aires de comienzos del siglo XX", Revista Educación y Pedagogía. Facultad de Educación, Universidad de Antioquia, Medellín, mayo-agosto, 22 (57), pp. 51-72. 